\title{
SECOND HARMONIC GENERATION FROM THIN SLABS IN THE DISCRETE DIPOLE APPROACH
}

\author{
C.M.J. Wijers \\ Faculty of Applied Science, Twente University of Technology, NL-7500 AE Enschede, The Netherlands \\ Th. Rasing \\ Research Institute for Materials, University of Nijmegen, Toernooiveld, \\ NL-6525 ED Nijmegen, The Netherlands \\ and \\ R.W.J. Hollering
}

Philips Research Laboratories, P.O. Box 80.000, 5600 JA Eindhoven, The Netherlands

(Received 20 March 1992 by D. Van Dyck)

\begin{abstract}
The nonlinear optical response of thin Si slabs is calculated using a discrete dipole approach. The $s$-polarized second harmonic response as a function of the angle of incidence appears to be in reasonable agreement with experimental results. The p-polarized SHG shows a high sensitivity for the shape of the polarizability profile.
\end{abstract}

RECENTLY nonlinear optical techniques like Second Harmonic Generation (SHG) and Sum Frequency Generation (SFG) have been proven to be versatile and sensitive surface and interface probes $[1,2]$. They have found application in such varying subjects as the study of the structure of interfacial molecular monolayers and electrodes $[1-3]$, the spectroscopy of buried interfaces [4] and the in situ monitoring of surface dynamics $[5,6]$ and epitaxial growth [7]. SHG arises from the nonlinear polarization $\mathbf{P}(2 \omega)$ induced by an incident laser field $\mathbf{E}(\omega)$ :

$\mathbf{P}(2 \omega)=\ddot{\boldsymbol{\chi}}^{(2)} \mathbf{E}(\omega) \mathbf{E}(\omega)$

where the nonlinear susceptibility $\chi^{(2)}$ contains material parameters.

For a centrosymmetric medium, $\boldsymbol{x}^{(2)}$ is only nonvanishing at symmetry breaking interfaces, which accounts for the surface sensitivity of SHG (and analogously of SFG). Most theoretical approaches to surface SHG are predominantly generalizations of bulk continuum descriptions [8] in combination with the nonlinear laws of reflection and refraction as derived by Bloembergen et al. [9]. Except for the time-dependent density functional approach by A. Liebsch [10], that describes the isotropic nonlinear response of simple (jellium like) metal surfaces and recent bandstructure calculations for $\mathrm{Si}-\mathrm{Ge}$ superlattices [11], no true microscopic calculations for the surface nonlinear optical response have been performed so far.

In this paper we will show how one can calculate the SHG from thin dielectric slabs by describing them with lattice planes of discrete, nonlinear dipoles. Such an approach is much better suited to incorporate the influence of local fields and generally yields a more flexible description of surface phenomena. In particular, the dependence on the angle of incidence will be studied. We will also investigate if such a model yields an adequate description of the nonlinear response of semiinfinite systems. The numerical results are compared with SHG data for $\mathrm{Si}$ and found to be in reasonable agreement. The method closely follows the equivalent method for the linear Surface Induced Optical Anisotropy (SIOA) problem, that was successfully developed earlier by us [12].

A thin dielectric slab, can be described by a set of characteristic dipoles $\mathbf{p}_{i}$ (located at $\mathbf{r}_{i}$ ), belonging to and completely governing the optical response of dipole lattice planes $i$. These $p_{i}$ can be obtained in the linear case by solving the slab interaction equations, given by:

$$
\begin{aligned}
& \mathbf{p}_{i}(\omega)=\sum_{j} \dot{\overrightarrow{\mathbf{M}}}_{i j}^{-1}(\omega) \mathbf{E}_{\mathrm{EXT}}\left(\mathbf{r}_{j}, \omega\right) \\
& \dot{\mathbf{M}}_{i j}(\omega)=\stackrel{\overrightarrow{\boldsymbol{\alpha}}}{i}_{i}^{-1} \delta_{i j}-\alpha_{0}^{-1} \vec{f}_{i j}
\end{aligned}
$$


where $\alpha_{i}$ represents the local polarizability tensor governing the $i$ th plane, $\alpha_{0}=4 \pi \epsilon_{0} \alpha^{3}, a=$ lattice constant and $f$ is a transfer tensor [12]. $\mathbf{E}_{\mathrm{EXT}}$ is the externally incident electric field of frequency $\omega$, wavevector $\mathbf{k}$ and amplitude $\mathbf{E}_{0}$. Once the $\mathbf{p}_{i}$ are known, the remote optical response of the slab can be exactly calculated.

We have shown that this scheme of calculation yields a highly flexible instrument to study the linear optical response of thin slabs, especially of the anisotropy involved. Within the basic assumptions of discrete dipole theory, the results obtained are exact.

We want to extend this model for nonlinear cases. The only modification concerns the induction principle, becoming in simplified form (time retardation omitted) for a separate dipole:

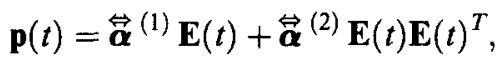

where $\mathbf{E}(t)$ is the instantaneous electrical field at the dipole.From this equation if follows, that the second harmonic obeys parallel translational symmetry in the same way as the fundamental frequency [12]:

$\mathbf{p}_{l}(\omega)=\mathbf{p}_{0}(\omega) \mathrm{e}^{i 2 \mathbf{k}_{\|}(\omega) \cdot \mathbf{s}_{l}}$

where the index $l$ refers to a general site within the lattice plane. From equation ( $3 b$ ) follows that the second harmonic reflection is specular and that transfer tensors can be used again to obtain the second harmonic electric fields radiated by the planes of dipoles. If beam depletion can be neglected, as is the case for SHG from surfaces of bulk centrosymmetric crystals, the nonlinear interaction equations acquire the following simple form:

$$
\begin{gathered}
\mathbf{p}_{i}(2 \omega)=\sum_{j} \dot{\mathbf{M}}_{i j}^{-1}(2 \omega) \mathbf{E}_{\mathrm{INH}}^{j}(2 \omega) \\
\mathbf{E}_{\mathrm{INH}}^{j}(2 \omega)=\left\{\dot{\boldsymbol{\alpha}}_{j}^{(1)}(2 \omega)\right\}^{-1} \hat{\boldsymbol{\alpha}}_{j}^{(2)}(2 \omega) \\
{\left[\left\{\hat{\boldsymbol{\alpha}}_{j}^{(1)}(\omega)\right\}^{-1} \mathbf{p}_{j}(\omega)\right]\left[\left\{\hat{\boldsymbol{\alpha}}_{j}^{(1)}(\omega)\right\}^{-1} \mathbf{p}_{j}(\omega)\right]^{T} .}
\end{gathered}
$$

For a slab of $N$ layers of dipoles equation (4a) results in a $3 N \times 3 N$ complex matrix problem, which can be treated in exactly the same way as for the SIOA linear problem. For the calculations, we take a slab of $N$ layers, where $N$ has been varied between 20 and 80 .
First $\mathbf{p}_{i}(\omega)$ is calculated from equation (2) following [12]. Full solution of the equation (4) finally yields the second harmonic dipole strength's $\mathbf{p}_{i}(2 \omega)$. The local and far away e.m. fields are calculated by superposition of the spherical waves emitted by the induced dipoles, and from that the nonlinear reflectivity can be obtained by:

$r_{t}=\frac{2 \pi i a^{3} k^{2}}{S_{\mathrm{WS}}\left|k_{z}\right| \alpha_{0} E_{0}}\left[\hat{\mathbf{t}} \cdot \sum_{j} \exp \left(i \mathbf{k} \cdot \mathbf{r}_{j}\right) \mathbf{p}_{j}\right]$.

$S_{\mathrm{WS}}$ is the area of the surface unit cell and $\hat{\mathbf{t}}$ the polarization vector of the reflected beam [12].

Comparing our calculations with a macroscopic [8-10] approach, one immediately realizes that in our case we do not have to apply boundary conditions to obtain the reflected e.m. fields. Similarly as for the linear case, this is a result of the fact that the local dipole strengths are calculated directly, whereas, the emitted electrical fields are then obtained by direct superposition. As an additional benefit, a high degree of freedom has been obtained to incorporate the surface microscopic geometry.

We will apply the method described above to a thin $\operatorname{Si}\left(\begin{array}{ll}1 & 10\end{array}\right)$ slab, composed of simple f.c.c. lattice planes of dipoles. The polarizabilities for the surface planes are obtained from [13] and the remaining bulk polarizabilities follow from the bulk dielectric constant via the Clausius-Mossotti relation. The intrinsic anisotropic surface properties are contained in the three outermost lattice planes [13]. The results are calculated for a fundamental Nd-YAG frequency of $1.064 \mu \mathrm{m}(1.15 \mathrm{eV})$. Table 1 summarizes the parameters in units of $\alpha_{0}=7.1328 \times 10^{-39} \mathrm{Fm}^{2}$. The anisotropic azimuth $\Omega$ is defined as the angle between the plane of incidence and the surface $(110)$ direction.

Figure 1 shows the results of the calculation of $p_{i}(2 \omega)$ for a slab of 32 layers.

Consistent with bulk inversion symmetry the hyperpolarizability $\alpha^{(2)}(2 \omega)$ has been chosen zero for the bulk. $\alpha_{\text {surf }}^{(2)}(2 \omega)$ had an isotropic value of $10^{-50} \mathrm{Fm}^{2}$ for the two surface regions and for the electric field strength $10^{7} \mathrm{~V} \mathrm{~m}^{-1}$ was used. The latter corresponds to a typical laser fluence of $5 \mathrm{~mJ} / \mathrm{mm}^{2}$ for a $10 \mathrm{~ns}$ pulse. Figure 1 shows that the outermost

\begin{tabular}{|c|c|c|}
\hline$\alpha$ & $\omega$ & $2 u$ \\
\hline 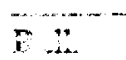 & $0.124073=00002525^{\circ}$ & $0.1342297+i 0.000703$ \\
\hline 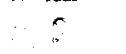 & $0.12425115-; 0.00 \mathrm{CLA}: 0 \mathrm{~A}$ & $0.1344456+i 0.00155 \epsilon$ \\
\hline$r$ & $0.1225435-i 0.000418^{n}$ & $0.132321 ?+i 0.001730$ \\
\hline
\end{tabular}

Table 1. Linear and nonlinear susceptibilities for a $\mathrm{Si}(110)$ surface at $\hbar \omega=1.15 \mathrm{eV}$. All values are in units of $\alpha_{\mathrm{f}}$. $\mathrm{x} \equiv 110, \mathrm{v} \equiv 0) 1$ 


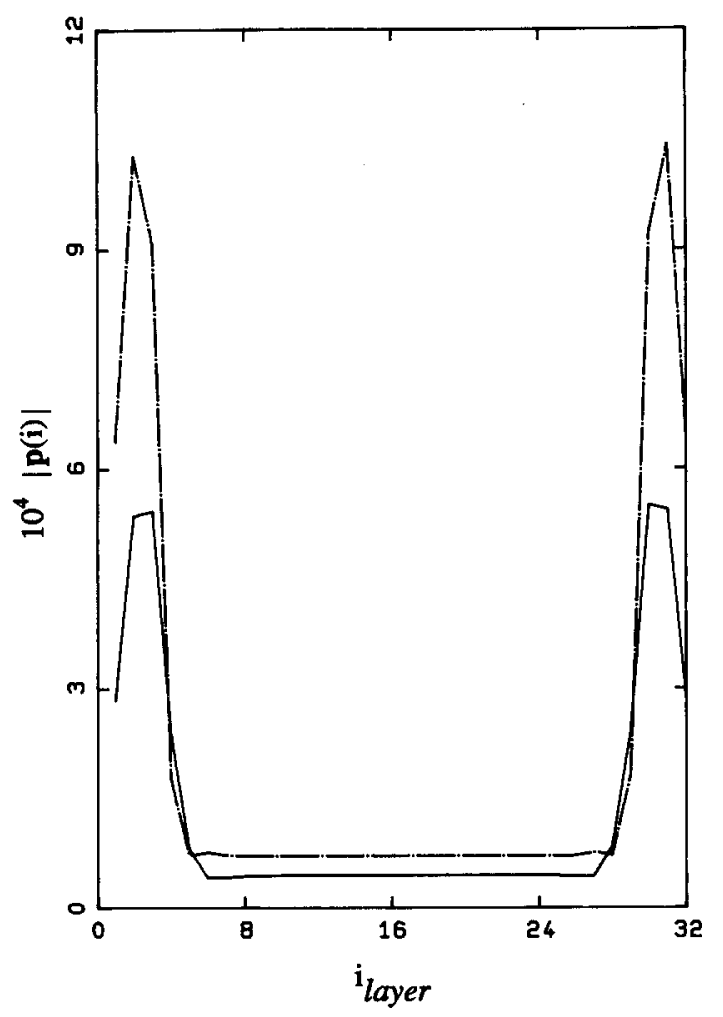

Fig. 1. Modulus $\left|\mathbf{p}_{i}(2)\right|$ as a function of layer index $i$ for a $\mathrm{Si}(110)$ slab at $\hbar \omega=1.15 \mathrm{eV}, \theta_{i}=0^{\circ}$ and \# layers $=32 .-: \Omega=0^{\circ}-\cdot-: \Omega=90^{\circ}$.

layers of the slab display a strong nonlinear response. In the slab interior an almost flat dipole strength can be observed, showing that bulklike conditions have been obtained. It is important to notice that the second harmonic response $p_{i}(2 \omega)$ is nonzero in this bulklike region, although $\alpha^{(2)}{ }_{i}(2 \omega)$ equals zero there. This is a result of the linear response in the bulk at $2 \omega$ caused by the surface emitted SHG fields.

This behaviour is contrary to the SHG continuum treatment, where (in the electric dipole approximation), the bulk nonlinear susceptibility and 2 nd harmonic sources disappear [9]. For higher frequencies (above about $3 \mathrm{eV}$ ) the strong nonlinear surface response $\mathbf{p}_{i}(2 \omega)$ will involve substantially more layers than three and will show an oscillatory behaviour [12].

From these values for $\mathbf{p}_{i}(2 \omega)$ the reflected SHG intensity for such a slab is calculated.

Figure 2 shows the angular dependence (normalized with respect to $\theta=0^{\circ}$ ) of the $s$-polarized SHG intensity for a $s$-polarized input beam. To indicate the convergence of the slab calculations we have plotted the results for a number of different layer thicknesses. Also plotted in Fig. 2 are the experimental data points for $\mathrm{Si}\left(\begin{array}{lll}1 & 1 & 1\end{array}\right)$ normalized in the same way. For

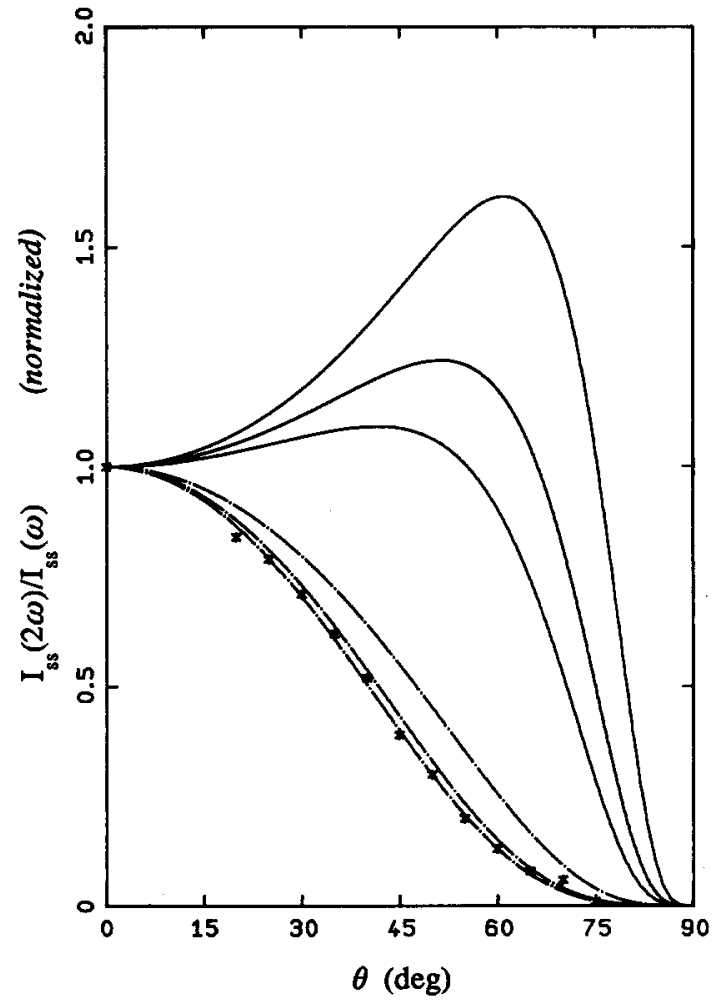

Fig. 2. Convergence behaviour: Normalized ss SHG reflection $I(2 \omega) / I(\omega)$ from a $\operatorname{Si}(110)$ slab as a function of the angle of incidence $\theta .-: \hbar \omega=1.15 \mathrm{eV}$, \# Layers: $50,65,79 ;-^{-}-: \hbar \omega=9.20 \mathrm{eV}$, \# Layers: 50 , 65, 79. Crosses: experiment.

the experiment we used the fundamental frequency of a Nd-YAG laser at $1.06 \mu \mathrm{m}(1.15 \mathrm{eV})$. The sample was an optically flat, standard oxidized $\mathrm{Si}\left(\begin{array}{lll}1 & 1 & 1\end{array}\right)$ wafer.

From the solid curves in Fig. 2 we learn that no convergence has been obtained yet for 50,65 and 79 layer slabs at $1.15 \mathrm{eV}$. In an artificial manner the convergence can be improved. We simply increase the frequency from $1.15 \mathrm{eV}$ to $9.2 \mathrm{eV}$ and leave all other parameters the same. (This procedure has been shown to be equivalent to increasing the slab thickness with a factor of 8 , but costs much less computing time.) For 79 layers and $9.2 \mathrm{eV}$ a very good agreement as compared to experiment can be obtained, although these results are still a few percent from full convergence. Moreover, the experimental data concern oxidized $\mathrm{Si}\left(\begin{array}{lll}1 & 1 & 1\end{array}\right)$, whereas, the calculation has been made for unreconstructed $\mathrm{Si}(110)$. So although the agreement has to a certain extent to be considered as fortuitous, the results of Fig. 2 contain a good indication, that a nonlinear discrete dipole calculation is suited to describe SHG.

From the linear continuum treatment of surface 


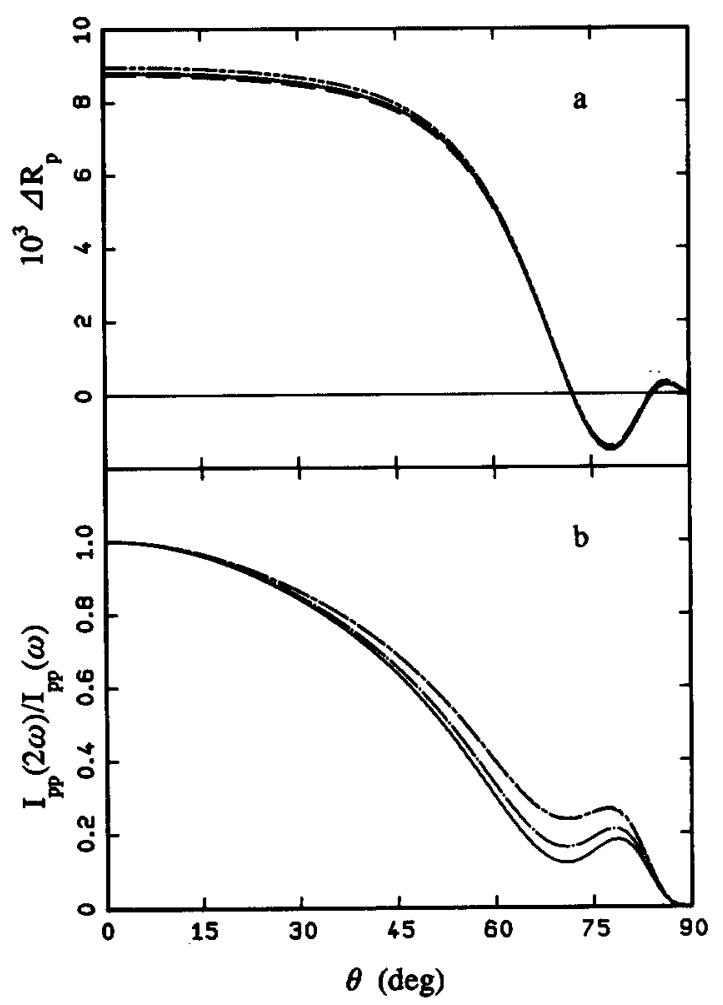

Fig. 3. Profile influence: $p$-type anisotropic reflectance difference $\Delta R$ and $S H G$ reflection from a $\mathrm{Si}(110)$ slab as a function of $\theta$. \# layers: 79 and $h \omega=9.2 \mathrm{eV}$. (a) $\Delta R$, (b) SHG. $r_{s}=3.0$ :-rectangular, ---: triangular, -- : hyperbolic. $r_{s}=6.0-\cdots-$ : rectangular.

optics, it is known that differential reflectance measurements yield only information about $\Delta \epsilon_{s s} d_{s}$, where $\Delta \epsilon_{s s}$, indicates the difference between the bulk dielectric constant and the dielectric constant of the surface layer (thickness $d_{s}$ ) [14]. Neither of these properties can be obtained independently, according to the McIntyre-Aspnes model which we have found to be in agreement with discrete dipole calculations. The discrete interpretation of the McIntyre-Aspnes constraint requires that the sum of the differences between layer polarizability and bulk polarizability has to be invariant. Figure 3(a) shows the the p-type anisotropic reflectance difference $\delta R$ for 4 different polarizability profiles obeying this constraint $\left(r_{s}\right.$ is the effective depth of the profile). The $\Delta R$ results display hardly any profile sensitivity. The nonlinear response for the same 4 cases is shown in Fig. 3(b). Now for the higher energy of $9.2 \mathrm{eV}$ a minimum comes out clearly near $70^{\circ}$ angle of incidence. The precise interpretation of this minimum requires a more detailed investigation (as we know now it does not behave like a Brewster minimum), but the pronounced dependency from the profile shape is clearly demonstrated. The possible implication from these few calculations is that from combined linear and nonlinear observations information can be obtained about the range of surface optical effects in the direction perpendicular to the surface.

In conclusion we have shown in this paper that the classical problem of SHG from bulk centrosymmetric systems can be solved exactly within the discrete dipole approach, using the solution of the related linear SIOA problem as a starting point. Different from the continuum picture, the discrete model predicts weak nonlinear bulk response. SHG and SIOA turn out to be closely related phenomena. Although the results given here are restricted to thin slabs only, the results obtained are already in reasonable agreement with experiments.

Compared to $s$-type SHG, an extra minimum in the $p$-type SHG has been found, as well as an increased sensitivity for the shape of the polarizability profile. All benefits which have been found for the discrete dipole model before, such as direct access to microscopy, influence of surface geometry and sensitivity for the outermost monolayers, have been found for the nonlinear case as well.

The rigorous discrete dipole approach also opens new roads for the quantitative description of SHG, as we hope to work out in future publications. In particular, the anisotropy of the SHG from crystalline semiconductor surfaces can be addressed directly in this way.

Acknowledgements - We would like to thank Prof. R. Del Sole and F. Manghi for letting us use their $\mathrm{Si}$ data. Part of this work has been supported by the Stichting voor Fundamenteel Onderzoek der Materie (FOM), which is financially supported by the Nederlandse Organisatie voor Wetenschappelijk Onderzoek (NWO).

\section{REFERENCES}

1. Y.R. Shen, Nature 337, 519 (1989).

2. G.L. Richmond, J.M. Robinson \& V.L. Shannon, Prog. Surf. Sci. 28, 1 (1988).

3. P. Goyot-Sionnest, a. Tadjedinne \& A. Liebsch, Phys. Rev. Lett. 64, 1678 (1990).

4. T.F. Heinz, F.J. Himpsel, E. Palange \& E. Burstein, Phys. Rev. Lett. 63, 644 (1989).

5. H.W.K. Tom, G.D. Aumiller \& C.H. BritoCruz, Phys. Rev. Lett. 60, 1438 (1988).

6. X.D. Zhu, Th. Rasing \& Y.R. Shen, Phys. Rev. Lett. 61, 2883 (1988).

7. R.W.J. Hollering, A.J. Hoeven \& J.M. Lenssinck, J. Vac. Sci. Technol. A8, 3194 (1990).

8. V. Mizrahi \& J.E. Sipe, J. Opt. Soc. B5, 660 (1988).

9. N. Bloembergen, Nonlinear Optics. Benjamin, New York (1965). 
10. A. Liebsch, Phys. Rev. Lett. 61, 1233 (1988).

11. E. Ghahramani, D.J. Moss \& J.E. Sipe, Phys. Rev. Lett. 64, 2815 (1990).

12. C.M.J. Wijers \& R. Del Sole, Physica Scripta T25, 325 (1989); C.M.J. Wijers \& G.P.M.
Poppe, Vacuum 41, 624 (1990).

13. C.M.J. Wijers, R. Del Sole \& F. Manghi, Phys. Rev. B44, 1825 (1991).

14. J.D.E. McIntyre \& D.E. Aspnes, Surf. Sci. 24, 417 (1971). 